(c) 2018 IEEE. Personal use of this material is permitted. Permission from IEEE must be obtained for all other uses, in any current or future media, including reprinting/republishing this material for advertising or promotional purposes, creating new collective works, for resale or redistribution to servers or lists, or reuse of any copyrighted component of this work in other works. 


\section{High Birefringent ENZ Photonic Crystal Fibers}

\author{
$1^{\text {st }}$ Tianyu Yang \\ Global Big Data Technolgoies Centre \\ University of Technolgoy Sydney \\ Sydney, Australia \\ tianyu.yang@student.uts.edu.au \\ $3^{\text {rd }}$ Richard W. Ziolkowski \\ Global Big Data Technolgoies Centre \\ University of Technolgoy Sydney \\ Sydney, Australia \\ Richard.Ziolkowski@uts.edu.au
}

\author{
$2^{\text {nd }}$ Can Ding \\ Global Big Data Technolgoies Centre \\ University of Technolgoy Sydney \\ Sydney, Australia \\ Can.Ding@uts.edu.au \\ $4^{\text {th }}$ Y. Jay Guo \\ Global Big Data Technolgoies Centre \\ University of Technolgoy Sydney \\ Sydney, Australia \\ Jay.Guo@uts.edu.au
}

\begin{abstract}
A novel photonic crystal fiber (PCF) design that has a simple circular air hole configuration is reported that yields a very high birefringence. The enhanced birefringence is achieved by filling a select number of the air holes in its cladding with an epsilon-near-zero (ENZ) material to break the index symmetry of its $\mathrm{X}$ - and $\mathrm{Y}$-polarization states. Comparisons of initial numerical simulations based on ideal ENZ materials and then those based on realistic ones demonstrate that the high birefringence property is still maintainable with currently available ENZ materials.
\end{abstract}

Keywords- Birefringence, fiber characterization, fiber materials, photonic crystal fiber (PCF), simulations

\section{INTRODUCTION}

Photonic crystal fibers (PCFs) have been successfully deployed in both the terahertz $(\mathrm{THz})$ and optical regimes. Their performance advantages, including good confinement, low loss, tunable dispersion, and structural flexibility [1-2], are desirable for many applications. Several key PCF characteristics, notably loss, birefringence, bandwidth and dispersion, must be considered for practical $\mathrm{THz}$ applications.

High birefringent PCFs are particularly useful for polarization-based $\mathrm{THz}$ guiding, sensing, filtering, and splitting applications. The most widely used method to introduce birefringence into a $\mathrm{THz}$ PCF is to geometrically break the symmetry within their holey claddings and/or core areas. For example, by introducing shaped air holes (elliptical [3], rectangular [4]) that have different effects on the X-and $\mathrm{Y}$-polarized fields propagating in it, one has a direct way to attain birefringence in a PCF. However, these intrinsically asymmetric holes are difficult to implement. Birefringence can also be obtained by employing asymmetric sets of circular holes [5], which are easier to fabricate. Nevertheless, they have not attained birefringence values as high as those based on the asymmetric rectangular or elliptical holes. The state-of-the-art birefringence even in those PCFs is limited to be lower than 0.1 .

In this paper, a THz PCF with very high birefringence values is developed which is constructed with only circular air holes. The high index contrast between the propagating X-and Y-polarized modes is realized by loading specific ones with materials having different refractive indexes $(n)$ to break the symmetry. In particular, holes in the first ring of the cladding are filled with either an epsilon-near-zero (ENZ) material $(n=0.1)$ or with air $(n=1.0)$. Additionally, the sizes of the air holes at different positions in the first ring are adjusted to further improve the index contrast by breaking the symmetry of the overall geometrical structure. The simulation results demonstrate that with ideal ENZ materials, the reported PCF design achieves a high birefringence near 0.3 , a low loss below $10^{-2} \mathrm{~cm}^{-1}$, and zero dispersion for frequencies around $0.75 \mathrm{THz}$. Moreover, when currently available, realistic ENZ materials are introduced into the PCF model, the simulation results demonstrate that good birefringence values are maintained but with an increase in the overall loss. Potential implementation techniques and hopes for lower loss ENZ materials are also discussed.

\section{PCF CONFIGURATION AND SIMULATIONS}

\section{A. Configuration}

Fig. 1 shows the cross section view of the high birefringent PCF. Its design is based on high resistivity silicon (HRS), which has a low bulk material absorption loss $<0.01 \mathrm{~cm}^{-1}$ from $0.1-1 \mathrm{THz}$ and has a stable refractive index $n=3.417$ in the frequency range of interest, from $0.5-4.0 \mathrm{THz}$ [6]. Five airhole rings with a triangular lattice distribution form its cladding. The two blue highlighted circular holes in the first ring have a diameter $d_{2}$. All of the rest of the circular holes have the diameter $d_{l}$. The distance between any two adjacent holes is the lattice constant $\Lambda$. A perfect matching layer whose thickness is $10 \%$ of the whole diameter of the PCF concentrically surrounds the hole-based cladding region. As shown in red in Fig. 1, four air holes are filled with an ENZ material. An ideal ENZ material is considered first. The real part of its refractive index is set to 0.1 and its imaginary part (material loss) is set to zero in the frequency range of interest. Realistic ENZ materials are then introduced for comparisons.

\section{B. Simulations}

The optimized PCF has $\Lambda=80 \mu \mathrm{m}, d_{1}=0.95 \Lambda$, and $d_{2}=$ $0.8 \Lambda$. Fig. 2 shows the simulated distributions of the magnitude of the electric field intensity for both its X-and Ypolarized modes. The field energy is well confined in the core region. Its simulated birefringence and total loss, TL (mainly

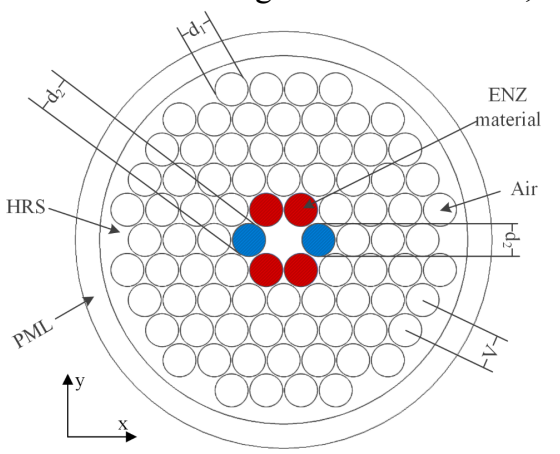

Fig. 1. Cross sectional view of the proposed PCF. 

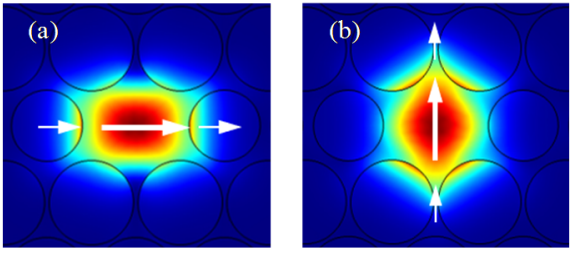

Fig. 2. Distributions of the magnitude of the electric field intensity distribution for the (a) X- and (b) Y-polarized modes. The white arrows designate the polarization direction. Blue represents low values; red represents high values.

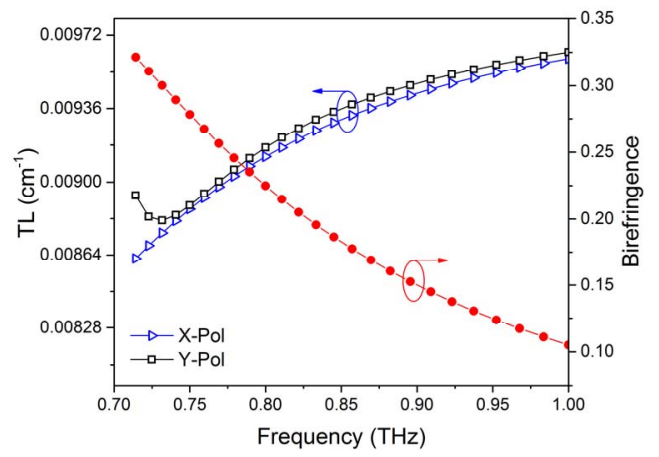

Fig. 3. Simulated performance characteristics of the optimized PCF as functions of the source frequency. Birefringence and TL values.

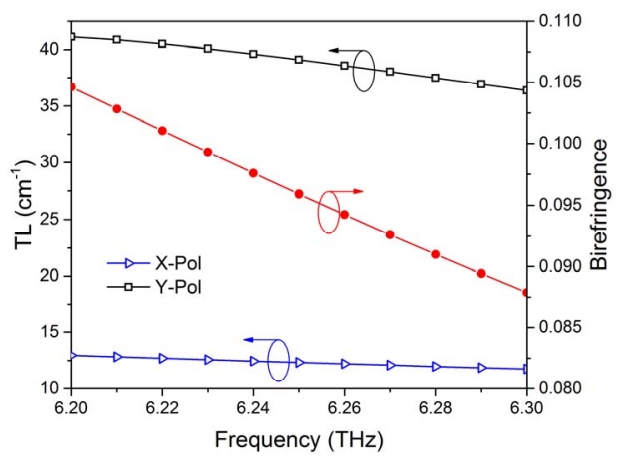

Fig. 4. Simulated performance characteristics of the realistic PCF as functions of the source frequency. Birefringence and TL values.

the confinement and effective material losses) values across the 0.71 to $1.0 \mathrm{THz}$ band are presented in Fig. 3. The birefringence values are high, above 0.1 across the entire band, with a maximum value of 0.32 at $0.71 \mathrm{THz}$. The corresponding TL values are below $0.01 \mathrm{~cm}^{-1}$. As will be shown in our presentation, the $\mathrm{X}$ - and Y-polarized propagating modes experience near zero dispersion with a very high birefringence value of 0.28 at $0.75 \mathrm{THz}$. The key factor to attaining these very high birefringence values is the presence of the ideal ENZ material in this optimized asymmetric configuration.

\section{III. "REALISTIC" PCF}

It was investigated to determine whether or not there are real or artificial materials that could provide the ideal ENZ properties. We have tried several known ENZ materials for our design. It was found that pure $\mathrm{KCl}$ in bulk form [7] is a good candidate for the $\mathrm{THz}$ PCF. Its ENZ crossover point occurs near 6.3 THz. With this much higher frequency ENZ crossover point, the background material had to be replaced. Topas was selected; it has a refractive index of $\mathrm{n}=1.53$ and an absorption loss of only $2.0 \mathrm{~cm}^{-1}$ near $6.3 \mathrm{THz}$ [8]. The PCF was re-optimized to this higher frequency with these materials and with the lattice period $\Lambda=25 \mu \mathrm{m}$. Its properties nicely scaled to this new operating frequency as was expected from the scaling principle reported in [2]. Fig. 4 shows the simulated birefringence and TL values of this realistic ENZ PCF. The birefringence value, 0.105 at $6.2 \mathrm{THz}$, is reasonable in comparison to the ideal value 0.28 and larger than most current PCFs. The TL values are $12.7 \mathrm{~cm}^{-1}$ and $41.3 \mathrm{~cm}^{-1}$ for the $\mathrm{X}$ - and Y-polarized modes, respectively.

Compared with the ideal case, the PCFs with realistic ENZ materials still achieve a relatively high birefringence. However, the TL values are much higher because the ENZ material losses are. Nonetheless, this first step of combining ENZ materials into a PCF design has shown quite interesting and useful performance characteristics. The real-world implementation of ENZ materials into structured fibers can be realized with hybrid optical fiber constructs [9] in several ways. For example, one could use the pressure-assisted melt filling technique [10] to insert the bulk-form of $\mathrm{KCl}$ [7] into the appropriate holes in a host silicon material for the $\mathrm{THz}$ PCF reported herein. We await the realization of lower loss ENZ materials before attempting any realistic PFC fabrication and measurement campaigns based on our designs.

\section{CONCLUSION}

This paper demonstrated a THz PCF design that achieves very high birefringence, low loss, and near zero dispersion characteristics with an ideal ENZ material. Realistic ENZ materials were also investigated. Comparisons between these two cases demonstrated that with currently available ENZ materials, high birefringence can still be obtained, but with a much higher loss. We anticipate that future realizations of ENZ materials will become available with much lower losses in the frequency bands of high interest.

\section{REFERENCES}

[1] P. St. J. Russell, "Photonic-Crystal Fibers," J. Lightw. Technol., vol. 24, no. 12 , pp. 4729-4749, 2006.

[2] T.-Y. Yang, C. Ding, R. W. Ziolkowski, and Y. J. Guo, “A scalable $\mathrm{THz}$ ultra-high birefringence and ultra-low loss partially-slotted photonic crystal fiber," IEEE J. Lightw. Technol., early access, DOI: 10.1109/JLT.2018.2842825, 2018.

[3] K. Ahmed et al., "Ultrahigh birefringence, ultralow material loss porous core single-mode fiber for terahertz wave guidance," Appl. Opt., vol. 56, no. 12, pp. 3477-3483, April 2017.

[4] S. Atakaramians et al., "THz porous fibers: design, fabrication and experimental characterization," Opt. Express, vol. 17, no. 16, pp. 14053-14062, Aug. 2009.

[5] T.-Y. Yang, E. Wang, H. Jiang, Z. Hu, and K. Xie, "High birefringence photonic crystal fiber with high nonlinearity and low confinement loss," Opt. Express, vol. 23, no. 7, pp. 8329-8337, April 2015.

[6] Dai, J. Q. Zhang, W. L. Zhang, and D. Grischkowsky, "Terahertz timedomain spectroscopy characterization of the far-infrared absorption and index of refraction of high-resistivity, float-zone silicon," Opt. Soc. Am. B, vol. 21, no. 7, pp. 1379-1386, 2004.

[7] A. Reyes-Coronado et al., "Self-organization approach for $\mathrm{THz}$ polaritonic metamaterials," Opt. Express, vol. 20, no. 13, pp. 1466314682, 2012.

[8] P. D. Cunningham et al., "Broadband terahertz characterization of the refractive index and absorption of some important polymeric and organic electro-optic materials," J. Appl. Phys., vol. 109, no. 4, pp. 043505, 2011.

[9] M. A. Schmidt, A. Argyros, and F. Sorin, "Hybrid optical fiber - an innovative platform for in-fiber photonic devices," Adv. Optical Mater., vol., 4, no., 1, pp. 13-36, 2016.

[10] H. W. Lee et al., "Pressure-assisted melt-filling and optical characterization of $\mathrm{Au}$ nano-wires in microstructured fibers," Opt. Express, vol. 19, no. 13, pp. 12180-12189, 2011. 